\title{
Large Eddy Simulation of the Stable Boundary-Layer: A Retrospect to Nieuwstadt's Early Work
}

\author{
Han van Dop • Simon Axelsen
}

Received: 16 June 2006 / Accepted: 29 March 2007 /

Published online: 23 August 2007

(C) Springer Science + Business Media B.V. 2007

\begin{abstract}
Observations and theory presented in a paper by Nieuwstadt ( $\mathrm{J}$ Atmos Sci 41:2202-2216, 1984) are reviewed and reconsidered. We have used a large eddy simulation (LES) model to make a 10-h rerun. Averaged results obtained for the last hour were considered to be representative for the wind-driven, quasi steady nocturnal boundary layer as reported in Nieuwstadt's paper. The turbulence characteristics found with the LES model are in good to excellent agreement with the majority of the observations and confirms the uniqueness of the dataset, though the scatter in the data is (understandably) large. Laboratory experiments of the stable boundary layer might reduce the uncertainty in existing data and should be encouraged. The concept of local scaling, introduced by Nieuwstadt in 1984 was also confirmed by our simulations. Nieuwstadt's experiment and local scaling theory of the SBL were a major achievement and an important contributions to our understanding of the SBL.
\end{abstract}

Keywords Stable boundary-layer $\cdot$ Large eddy simulation

\section{Introduction}

Theory and observations of the stable atmospheric boundary-layer (SBL) started to develop in the late seventies. Jean-Claude André, Julian Hunt, John Wyngaard, John Garratt, Larry Mahrt and Serge Zilitinkevich made significant contributions to the progress in understanding the dynamics and physics of the SBL. To this shortlist the

H. van Dop $(\varangle) \cdot$ S. Axelsen

IMAU, Utrecht University, P.O. Box 80.005,

3508 TA Utrecht, The Netherlands

e-mail: h.vandop@phys.uu.nl 
name of Frans Nieuwstadt should certainly be added. He introduced the concept of 'local scaling' in the SBL, which is the hypothesis that its (dimensionless) turbulence characteristics can be expressed as functions of the single parameter $z / \Lambda$, were $z$ is the height above the surface and $\Lambda(z)$ the 'local Obukhov length', expressed as

$$
\Lambda=-\frac{\tau^{3 / 2}}{\kappa(g / T) \overline{w \theta}} .
$$

The local stress and heat flux are denoted by $\tau$ and $\overline{w \theta}$, respectively. His second important contribution consisted of a series of nocturnal boundary-layer experiments (1977-1979) which resulted in (still!) one of the few available data sets containing detailed mean and turbulence characteristics of the quasi stationary SBL. His main findings were reported in [13], a paper to which we shall refer to as N84 hereafter.

Compared with the convective boundary layer (CBL), theory and certainly observations in the SBL are rather more complex. Shear is the only turbulence production mechanism, counteracted by buoyancy forces, which results in generally low turbulence levels, or in very stable conditions, to intermittent turbulence. Also, the stable stratification facilitates the generation of gravity waves which 'contaminate' the turbulence observations. Other complicating factors are (long wave) radiative effects and the occurrence of drainage or katabatic flow in SBLs over (even gentle) topography.

Theoretical studies of the SBL at that time were usually based on the Reynolds Averaged Navier-stokes (RANS) equations, see e.g. [6, 22]. Large-eddy simulations emerged more than a decade later $[1,12]$. Recently a group of numerical modellers performed an LES intercomparison study of the SBL $[4,10]$ in the framework of the Global Energy and Water Cycle Experiment (GEWEX) Atmospheric boundary layer Study (GABLS). In attempts to validate these findings, the data in N84 appeared to be very useful [3,12]. Basu et al. [3] used their LES results to review theory and observations in N84.

Although today's computers are much more powerful, LES studies of the SBL are still difficult which partly lies in the fact that the eddies are small and hence it is numerically difficult to keep the flow turbulent in relatively coarse grids.

We are currently employing an LES model with a view to apply it to an SBL over a downward tilted slope (katabatic flow). As a first step we have adapted a version of an LES model for a cloud-capped CBL (see [20]), in order to simulate a horizontally homogeneous SBL, driven by a constant pressure forcing and cooled at the surface in moderately stable conditions. Before embarking on katabatic flow, however, we had to verify that the model performs well in horizontally homogeneous conditions. We have investigated properties of the sub-grid scale (SGS) parameterization, grid size dependency and impact of numerical diffusion of the LES model. This is work in progress which will be reported in the near future. Nevertheless we obtained some interesting results which asked for a comparison with the data in N84 and in which we found an appropriate occasion to write this memorial paper.

We shall first briefly introduce the LES model used in this study and present the main results. Then we summarize the main findings in N84 and we will make a comparison between observed data and theory in N84 and our LES results. We shall also investigate to what extent our LES results are consistent with the local scaling hypothesis in N84.

을 Springer 


\section{The LES Model}

The equations for the evolution of the filtered velocity field are derived from the Navier-Stokes equations for incompressible flow in a geophysical frame of reference.The filtered conservation equations for momentum, heat and mass read, respectively

$$
\begin{aligned}
\frac{\partial \overline{u_{i}}}{\partial t}+\frac{\partial \overline{u_{i}} \overline{u_{j}}}{\partial x_{j}} & =-\frac{\partial \pi}{\partial x_{j}}-2 \epsilon_{i j k} \Omega_{j} \overline{u_{k}}+\frac{\bar{\theta}}{T} \delta_{i 3} g-\frac{\partial \tau_{i j}}{\partial x_{j}} \\
\frac{\partial \bar{\theta}}{\partial t}+\frac{\partial \bar{\theta} \overline{u_{j}}}{\partial x_{j}} & =\frac{\partial \tau_{\theta_{j}}}{\partial x_{j}} \\
\frac{\partial \overline{u_{i}}}{\partial x_{i}} & =0
\end{aligned}
$$

where

$$
\begin{gathered}
\pi=\frac{\bar{p}}{\rho_{0}}+\frac{2}{3} e \quad, \quad \tau_{i j}=\overline{u_{i} u_{j}}-\overline{u_{i}} \overline{u_{j}}-\frac{2}{3} e \delta_{i j} \\
\tau_{\theta_{j}}=\overline{u_{j} \theta}-\overline{u_{j}} \bar{\theta} \quad \text { and } \quad e=\frac{1}{2}\left(\overline{u_{i}^{2}}-{\overline{u_{i}}}^{2}\right) .
\end{gathered}
$$

The turbulent kinetic energy (TKE) of the unresolved eddies is denoted by $e, \pi$ is the modified pressure, $\tau_{i j}$ the residual stress tensor and $\tau_{\theta j}$ the residual heat flux. Molecular viscosity is neglected in (1). The Earth rotation vector is $\Omega_{j}$.

The residual fluxes are modelled as

$$
\tau_{i j}=-2 K_{m} \bar{S}_{i j} \quad \text { and } \quad \tau_{\theta j}=-K_{h} \frac{\partial \bar{\theta}}{\partial x_{j}},
$$

where

$$
\bar{S}_{i j}=\frac{1}{2}\left(\frac{\partial \overline{u_{i}}}{\partial x_{j}}+\frac{\partial \overline{u_{j}}}{\partial x_{i}}\right) .
$$

The eddy viscosities for momentum and heat $K_{m, h}$, are modelled according to $K_{m, h}=$ $c_{m, h} \lambda e^{\frac{1}{2}}$, where $\lambda$ is the filter width.

The residual TKE is obtained from the prognostic equation for $e$ :

$$
\frac{\mathrm{d} e}{\mathrm{~d} t}=\underbrace{K_{m} \frac{\partial \overline{u_{i}}}{\partial x_{j}}\left(\frac{\partial \overline{u_{i}}}{\partial x_{j}}+\frac{\partial \overline{u_{j}}}{\partial x_{i}}\right)}_{\text {Shear }}-\underbrace{K_{h} \frac{g}{T_{0}} \frac{\partial \bar{\theta}}{\partial z}}_{\text {Buoyancy }}+\underbrace{\frac{\partial}{\partial x_{j}} K_{m} \frac{\partial e}{\partial x_{j}}}_{\text {Transport }}-\underbrace{c_{\epsilon} \frac{e^{3 / 2}}{\lambda}}_{\text {Dissipation }},
$$

where $c_{\epsilon}$ is another constant. Assuming that the filter width lies within the inertial sub-range, we are able to derive appropriate values for the constants $c_{m, h, \epsilon}$ (see [23]).

In the (staggered equidistant) numerical discretisation of the advection terms a second order accurate scheme was used [17]. Diffusion terms are discretisized using second order central differences. The time integration of advection terms is done using a leap-frog method with an Asselin filter to avoid time splitting [2]. For the diffusion terms an Euler forward scheme was applied. The Poisson equation for the pressure was solved using the Fast Fourier Transform algorithm in the horizontal 
directions using cyclic boundary conditions, and a tridiagonal solver in the vertical [19]. A sponge layer, filling the top $25 \%$ of the grid prevents reflection of gravity waves at the model top.

An initial uniform wind field of $8 \mathrm{~m} \mathrm{~s}^{-1}$ (equal to the geostrophic wind) in the $\mathrm{x}$-direction was chosen The initial potential temperature was taken constant between 0 and $100 \mathrm{~m}$. Above $100 \mathrm{~m}$ a constant stable lapse rate of $0.01 \mathrm{~K} \mathrm{~m}^{-1}$ was assumed. At the surface a linear temperature decrease is prescribed of $-0.25 \mathrm{~K} \mathrm{~h}^{-1}$ which corresponded to an equilibrium heat flux of approximately $15 \mathrm{~W} \mathrm{~m}^{-2}$. Surface roughness was $0.1 \mathrm{~m}$. Runs were started with small random disturbances in the temperature fields and lasted for $10 \mathrm{~h}$. Results show the slab-average values of the last hour. Most of these data were taken from the model intercomparison study [5]. Finally, the domain size is $400 \mathrm{~m}$ in all three spatial directions.

So far the model is fairly standard. When running the model in stable conditions, grid spacing and time steps were reduced. Applying too coarse grids resulted in nonturbulent simulations. This could be avoided by artificially decreasing $\lambda$. This results in a larger dissipation term in (5) so that the equilibrium value of $e$ decreases. It results in smaller values for $K_{m, h}$ and thus in less damping of the resolved motion. Though it leads to 'turbulent runs' even for grid spacings as large as $12 \mathrm{~m}$, there is no justification for this adaptation. It leads to filter scales which become smaller than the grid spacing, which is already questionable and moreover, one may wonder what kind of results can be expected from the simulation of a turbulent flow where the grid spacing is of the same order of the typical length scales of stable turbulence. Let's consider the following arguments in selecting filter scale and grid spacing. In Fig. 1 we have heuristically indicated in a 1-D Kolmogorov spectrum the relevant length scales $\lambda_{m}$, corresponding with the spectral maximum, the filter scale $\lambda$ and the grid spacing $\Delta$. For a consistent estimate of the coefficients $c_{m, h, \epsilon}$ it is required that $\lambda$ lies in the inertial sub-range. This also guarantees that a substantial part of the turbulent

Fig. 1 A Kolmogorov spectrum representative for stable turbulence. Here, $L$ denotes the dissipation length scale, $R e_{\lambda}$ the Taylor-scale Reynolds number and $e$ the turbulent kinetic energy. Length scales $(l)$ and wavenumbers $(k)$ are related through $l=\pi / k$. Note that the horizontal axis is logarithmic with base 2

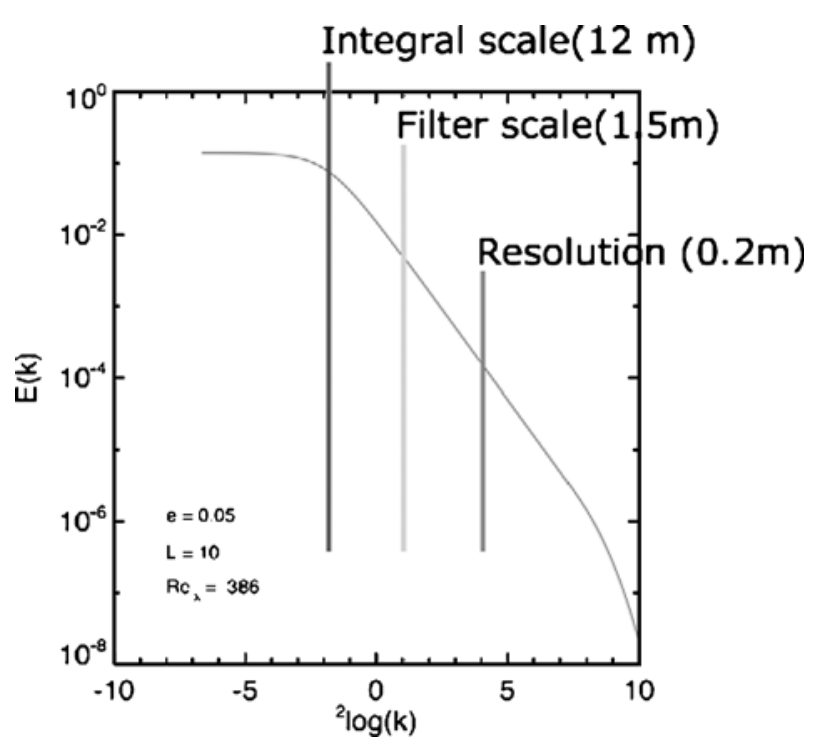


energy is resolved by the model, since $\lambda \ll \lambda_{m}$. Only when the grid spacing is smaller than the filter scale one may hope to obtain grid size independent simulations, a basic requirement in numerical modelling, thus

$$
\Delta \ll \lambda \ll \lambda_{m},
$$

as Fig. 1 suggests.

It is reasonable to assume that typical turbulence length scales based on stable turbulent spectra, velocity gradients or Brunt-Vaàisàlà frequency in the SBL are of the order of 10-15 m [7,11]. Appropriate choices for filter scales and grid spacing are indicated in the figure. We realise that in reality often compromises will be made in order to keep computer time managable. Our experience is also that a large variety of different SGS parameterizations do not produce widely different results. This is also confirmed by Beare, R. J. et al. This issue, including the role of numerical diffusion, will be pursued further in a companion paper. In the scope of this article we have chosen for a $64^{3}$ simulations (grid size $\sim 6 \mathrm{~m}$ ). The timestep compliant with the CFL criterion is $0.4 \mathrm{~s}$. We have also chosen to ignore the SGS parameterization for the reason given above. We have used simply $K_{m}=K_{h}=0.1 \mathrm{~m}^{2} \mathrm{~s}^{-1}$, thus constant values for the eddy diffusivity coefficients. It appears that for this resolution the model performs at least as good as in any other run with SGS parameterization. We shall address this issue extensively in the companion paper.

Finally we show the result of the resolved turbulent kinetic energy budget (Fig. 4). Away from the surface the transport terms ('transport' and 'prscorr') are small. The buoyancy term is important below, say $50 \mathrm{~m}$. The Figure basically suggest a balance between the dissipation and the shear production. Note that the residual term equals approximately half the dissipation term. We think that this term is the result of numerical errors (spurious diffusion) in the discretization scheme of the advection algorithm of the model. We shall not pursue this (important) issue here and we hope to report this in the near future.

\section{Results}

\subsection{Mean characteristics}

We shall first show some general results from the LES run. In Fig. 2 we see that within a couple of hours surface characteristics reach their equilibrium value. An Obukhov length of $102 \mathrm{~m}$ and a friction velocity of $0.26 \mathrm{~m} \mathrm{~s}^{-1}$ represent a moderately stable wind-driven SBL. The SBL height was inferred from $z_{0.05}$, the height where the stress attains $5 \%$ of its surface value. Using the relationship from N84,

$$
\frac{\tau}{\tau_{0}}=\left(1-\frac{z}{h}\right)^{\frac{3}{2}},
$$

we get

$$
h=\frac{z_{0.05}}{1-0.05^{\frac{2}{3}}},
$$


Fig. 2 Mean characterisitics of the model run for a $10 \mathrm{~h}$ simulation. After 5-6 h all paremeters are more or less stationary. The friction velocity is $u_{\star}$, the Obukhov length $L$, the kinematic heat flux at the surface $\overline{\theta w}$ and the rotation of the wind vector in the boundary layer is $\gamma$
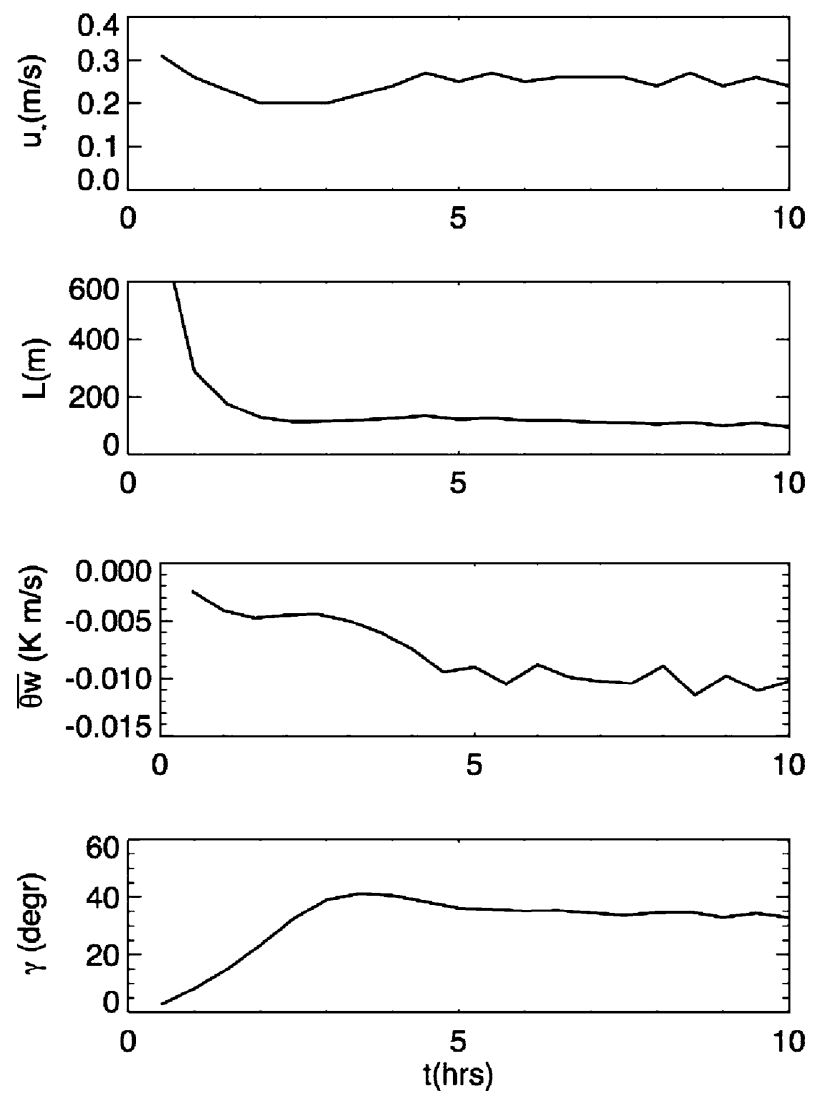

with the result $h=184 \mathrm{~m}$. (Note that this SBL definition slightly differs from the usual linear interpolation, see e.g. [5]). This value is reasonably in line with the stationary relationship

$$
h=c\left(\tau_{0}^{\frac{1}{2}} L /|f|\right)^{\frac{1}{2}}
$$

[9]. Our value for $h$ yields for the constant of proportionality 0.32 , whereas other studies suggest $c \approx 0.4$. The cross-isobar flow angle is $33^{\circ}$, well within the observed range in the SBL over a terrain roughness of $0.1 \mathrm{~m}$. The surface cooling of $0.25 \mathrm{~K} \mathrm{~h}^{-1}$ corresponds to an equilibrium heat flux of $-0.012 \mathrm{~K} \mathrm{~m} \mathrm{~s}^{-1}$.

In Fig. 3 mean wind and potential temperature are depicted. They are well within the range of model data in the framework of the SBL intercomparison study, [5]. Our temperature profile is very similar to the one used in N84, which is an interpolated composite of temperature profiles. Both profiles show a positive curvature $\left(d^{2} \Theta / d z^{2}>0\right)$ in almost the whole SBL, which is typical for a shear-driven weakly SBL.

Since the LES includes Coriolis forces, the wind profile has a maximum due to the inertial oscillation. This maximum is not present in the N84 data. This might be ascribed to the interpolation process in N84 where observations of more than 

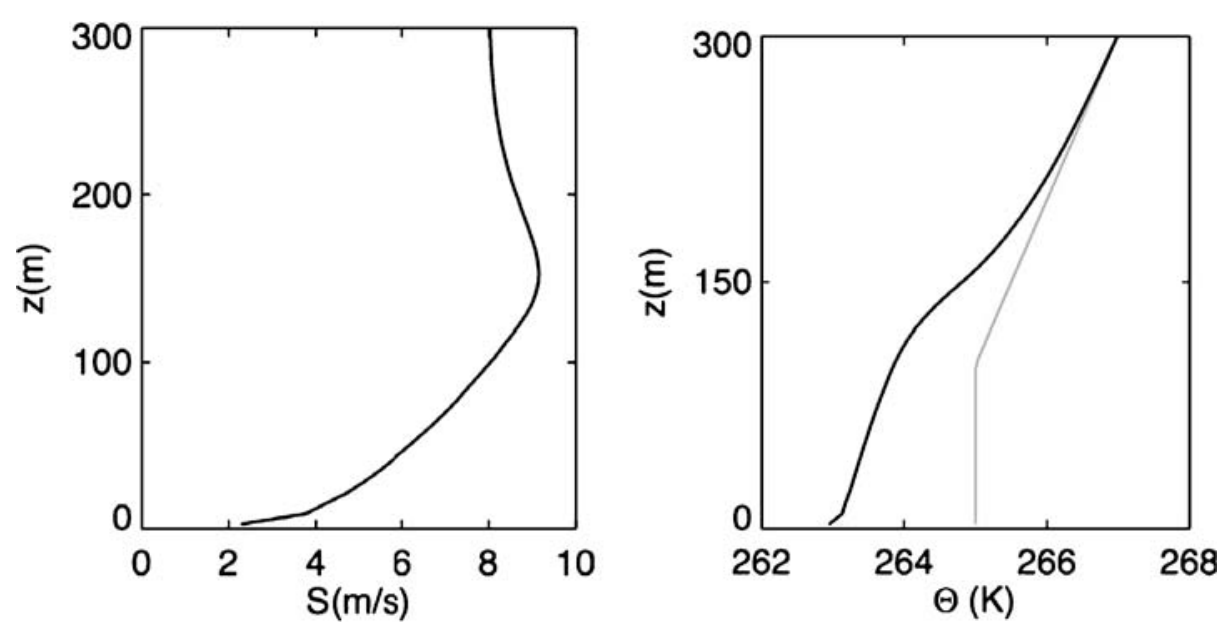

Fig. 3 The wind speed $S$ (left) and the potential temperature $(\Theta)$ as a function of height (right), averaged over the last simulation hour. The thin solid line in the latter plot is the initial temperature profile

100 profiles were used to construct a composite (z-dependent) wind profile, whereas individual profiles may have shown nocturnal jets which may be smoothed in the averaging process.

Observed boundary layer heights in N84 varied between 70 and $420 \mathrm{~m}$ with an average of $198 \mathrm{~m}$. Friction velocities ranged from 0.10 to $0.80 \mathrm{~m} \mathrm{~s}^{-1}$ (average 0.42 )

Fig. 4 Magnitude of the various terms in the resolved turbulent kinetic energy equation, in approximate stationary equilibrium (final hour). The (divergence of) turbulent transport and the pressure-velocity term are denoted by transport and prscorr, respectively. The vertical axis is the height (m). The residual dissipation completes the balance and is due to numerical diffusion

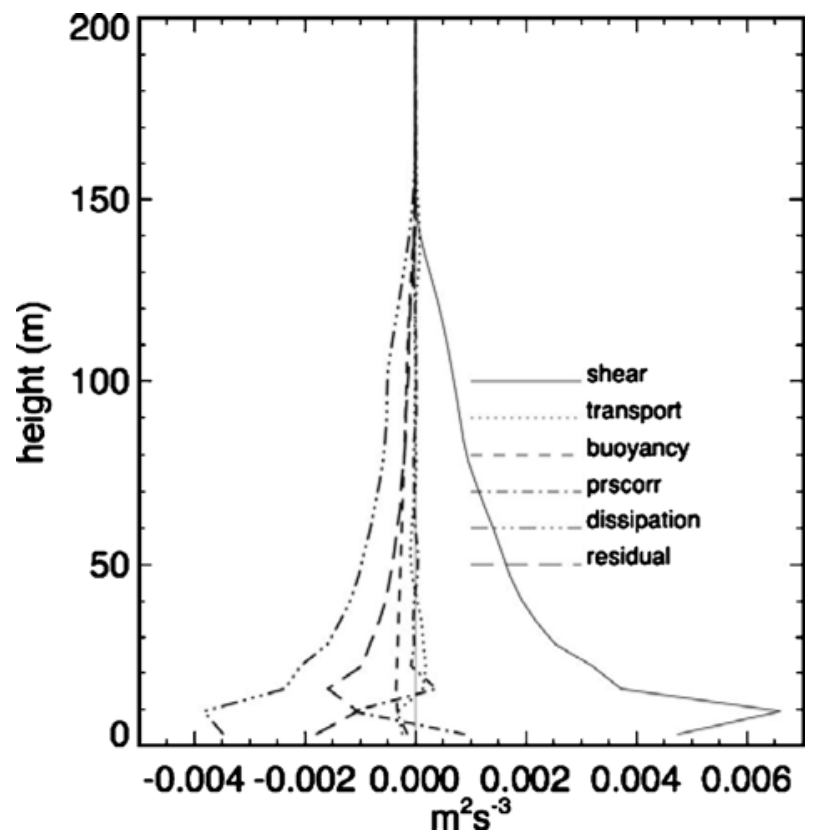


and surface heat fluxes from -0.003 to $-0.05 \mathrm{~K} \mathrm{~m} \mathrm{~s}^{-1}$ (average -0.018). The geostrophic wind varied between 4.7 and $22.2 \mathrm{~m} \mathrm{~s}^{-1}$ (average 11.6) and finally the stability parameter $h / L$ ranged from 0.2 to 6.5 (average 1.5 ). The corresponding value in our LES was 1.8 and thus very close to the observed average. We conclude that the LES profiles of mean temperature and wind speed are close to the corresponding averages in $\mathrm{N} 84$ and well within the range of observations used there.

\subsection{Turbulence profiles}

Since local scaling implies the knowledge of the vertical distribution of momentum and heat flux, we shall first present these data. Figure 5 shows the normalized heat flux as a function of $z / h$. The required linear relationship is present in both the observations and the LES. Note the large scatter in the observations partly due to the (still) notoriously difficult measurement of small turbulent heat fluxes in the SBL.

Figure 6 shows the normalized stress. There is an excellent agreement between LES and the theoretical prediction in N84 (6). The best fit to the LES data is obtained for the power 1.43 , which is very close to the theoretical value of $3 / 2$. Also the N84 observations are confirmative, though also here the scatter is large.

Since the observations and prediction in N84 and our LES result (see Fig. 9) indicate that $\sigma_{w} / \tau^{\frac{1}{2}}$ appears to be approximately constant, we find that the LES prediction, using (6), obeys $\sigma_{w} \propto\left(1-\frac{z}{h}\right)^{\frac{3}{4}}$.

Fig. 5 LES simulation (dashed line) and N84 data of the heat flux as a function of height. The asterisks denote the average values of the Cabauw observations and the grey area indicates the standard deviation. The solid line is the theoretical relationship

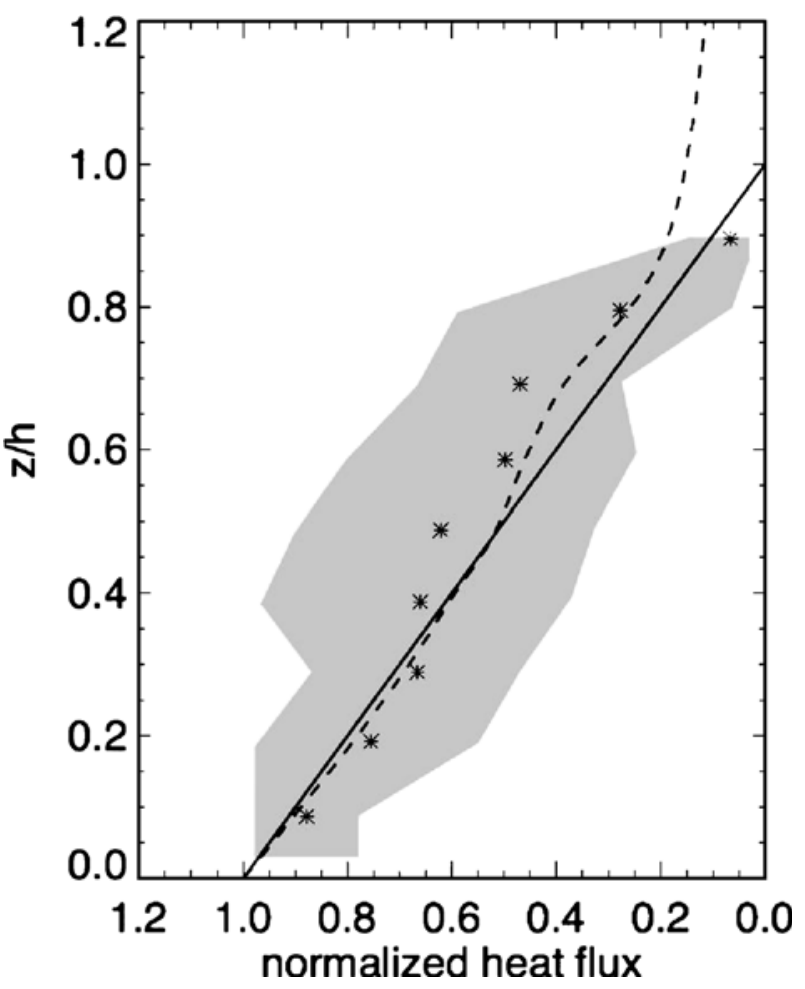


Fig. 6 Stress as a function of height. The notation is as in Fig. 5. The theoretical relationship is $\tau / u_{\star}^{2}=(1-z / h)^{\frac{3}{2}}$

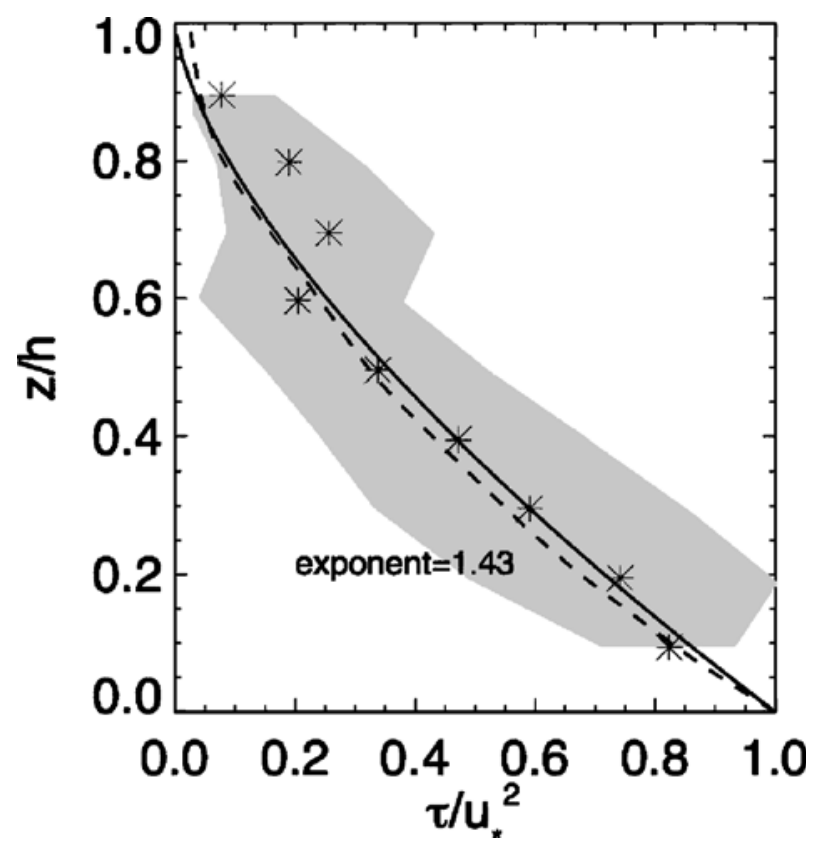

The result for the temperature variance is less convincing (see Fig. 7). In the original figure also the Minnesota were plotted which added even more discrepancy: only near the surface they are in the range five to seven and close to the other observations and predictions, but at higher elevation they soon decrease beyond two. The LES data are within the (large) measurement errors, but the average observations are much higher than the LES prediction. Finally the theoretical prediction looks essentially different, but that can be partly explained, as we shall shortly see.

Fig. 7 Temperature variance as a function of height. The notation is as in Fig. 5. In addition, the triangles represent the average values of the filtered observations (see the text)

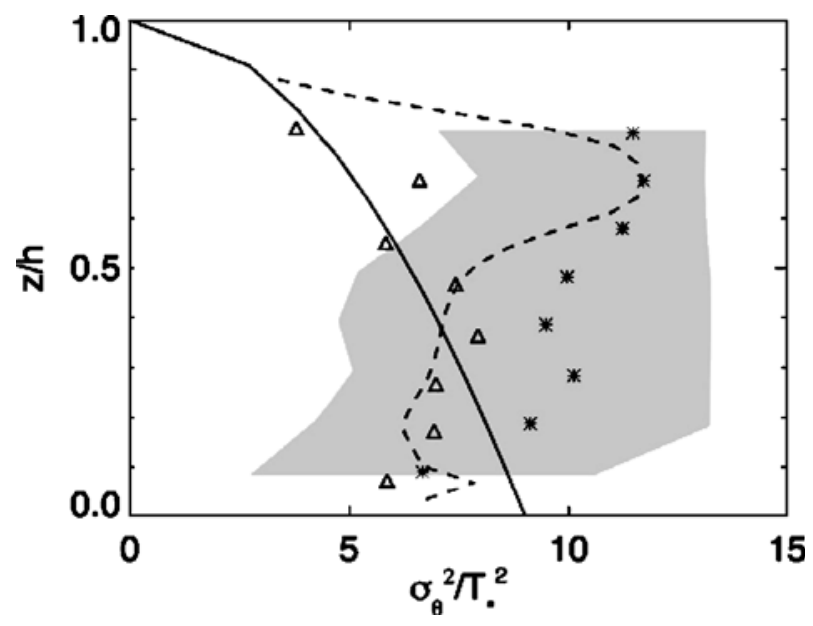


This is the only graph where we also show the average temperature variance for the filtered data (triangles). The reason is obvious, they are very close to the LES data up to $z / h=0.5$ and both tend to decrease similarly in the upper half of the SBL. From the original data in N84, a second data set was generated by Nieuwstadt, where low frequency wave motion was excluded from the time series by applying a high-pass filter with a cut-off frequency of $0.01 \mathrm{~Hz}$. This apparently leads to smaller temperature variances and better agreement with the LES prediction, suggesting that the data had been 'contaminated' with wave motion. We have no reason to assume that in the LES gravity waves are significant. In the model formulation a damping algorithm is active in the upper one quarter of the domain which filters out gravity waves. Note that the N84 prediction is also in better agreement with the filtered data.

\subsection{Local scaling}

One of the major questions in N84 was whether observed, properly scaled, turbulence data would be a function of $z / \Lambda$ only. This question was succesfully answered and confirmed the hypothesis based on the stationary solution of the second order variance and covariance equations. Here we shall review a selection of the N84 results and compare them with the results obtained with the LES model.

We start with a test of the generalized Monin-Obukhov relationship which e.g., for momentum would be

$$
\phi_{m}=1+5 z / \Lambda
$$

Fig. 8 The dimensionless velocity profile $\left(\phi_{m}\right)$ as a function of dimensionless height $(z / \Lambda)$. The notation is as in Fig. 5. The solid line represents the relationship $\phi_{m}=1+5 z / \Lambda$

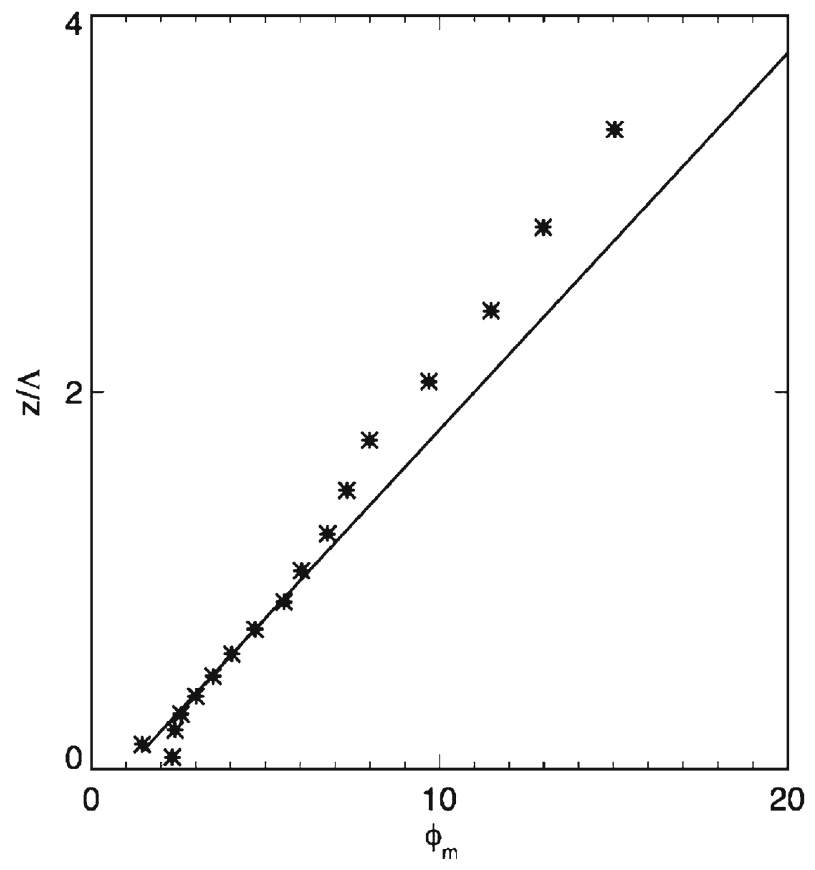


Fig. 9 Dimensionless vertical velocity variance as a function of $z / \Lambda$. The notation is as in Fig. 5

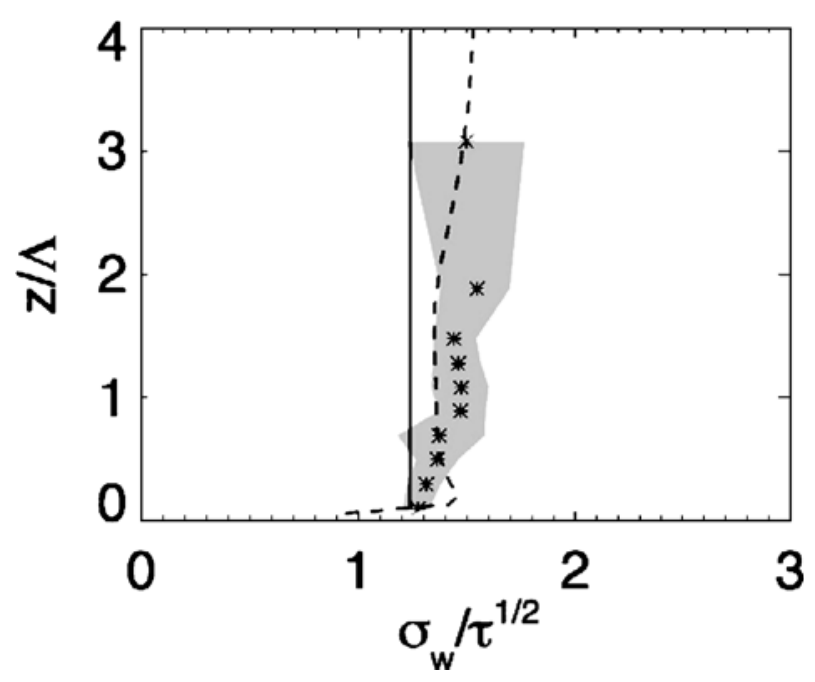

From Fig. 8 we conclude that the fit of the LES data is good up to $z / \Lambda \sim 1$. The fit with the slope 4.1 instead of 5 would be excellent up to $z / \Lambda \sim 5$. It should be noted, however, that in LES with an active SGS model fits may appear to be worse.

In Fig. 9 we see that the N84 prediction of the vertical velocity variance is slightly lower than the observation at all heights. Here the LES prediction is better with a z-less scaling limit $(z / \Lambda \rightarrow \infty)$ of $\sim 1.4$.

Fig. 10 Dimensionless temperature variance as a function of $z / \Lambda$. The notation is as in Fig. 5

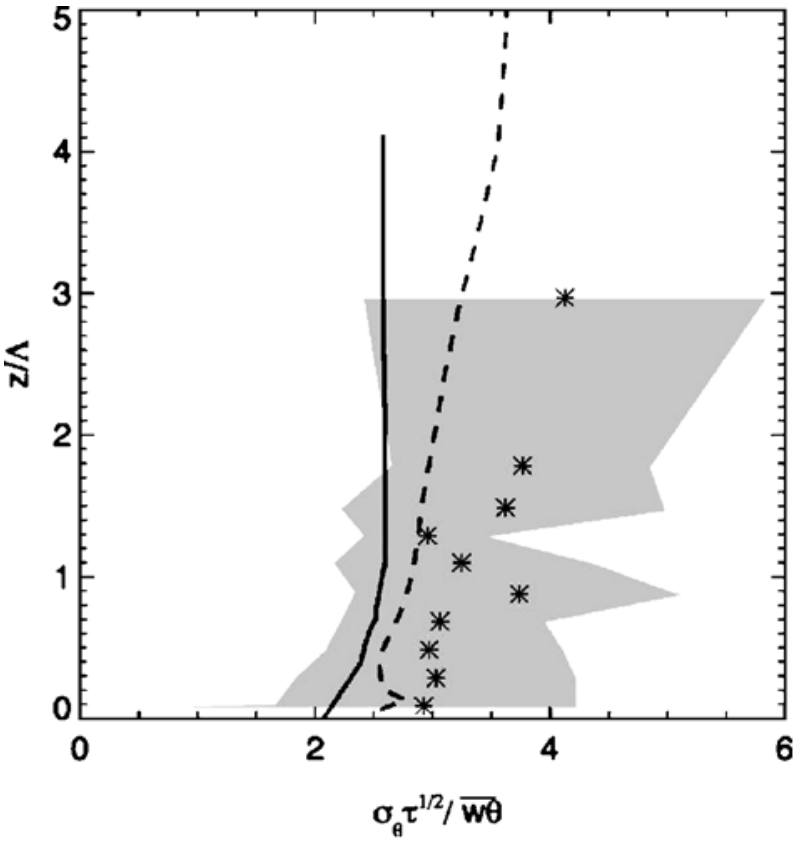



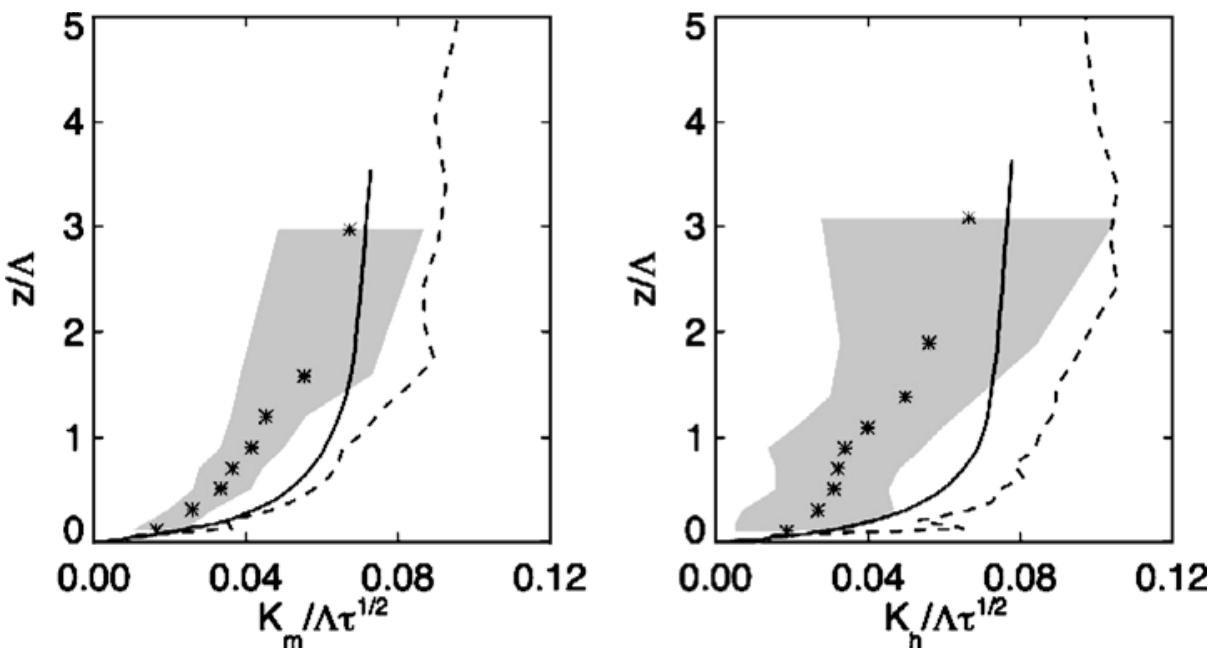

Fig. 11 Dimensionless profiles of the eddy viscosties for momentum (left) and heat (right). The notation is as in Fig. 5

The temperature variance is shown in Fig. 10. Also here the LES predictions are closer to the data and show a slight increase with height, similar to the observations. The LES z-less limit is $\sim 3.6$ compared to $\sim 3$ for the N84 prediction.

In Fig. 11 we see that the N84 and LES predictions are similar, especially for $z / \Lambda \lesssim 1$. They both overestimate the observations strongly though N84 less for larger values of $z / \Lambda$. In N84 the ratio $K_{m} / K_{h}$ is order 1 where the LES prediction yields values between 0.6 and 1.2. In this context it should be noted that $K_{m}$ and $K_{h}$ stand for the gradient-flux ratios $\frac{d U}{d z} / \overline{u w}$ and $\frac{d \Theta}{d z} / \overline{\theta w}$, respectively. Our LES results are well within the margins of the intercomparison study [4].

Fig. 12 The Richardson number as a function of $z / \Lambda$. The notation is as in Fig. 5

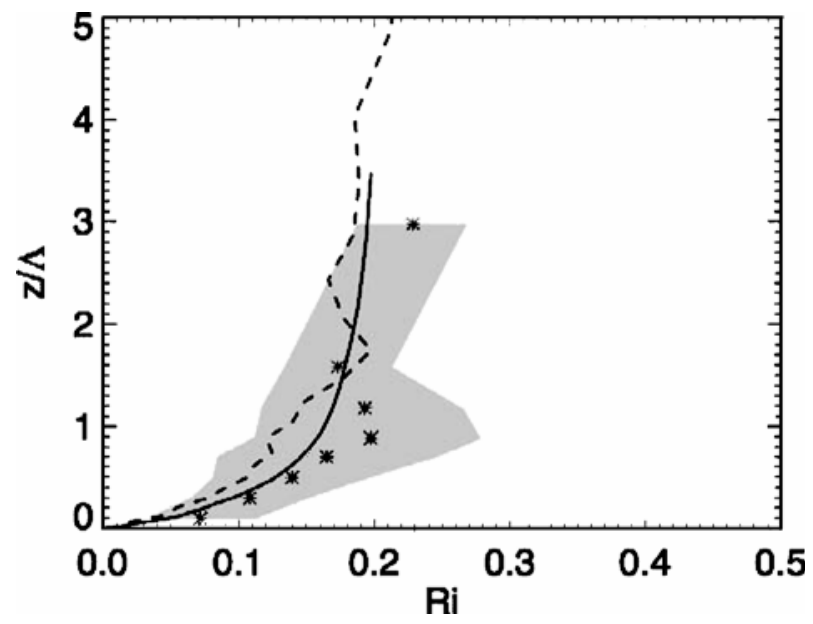


Finally we show in Fig. 12 the gradient Richardson number $R i$, defined as

$$
R i=\frac{\frac{g}{T} \frac{\partial \bar{\theta}}{\partial z}}{\left(\frac{\partial \bar{u}}{\partial z}\right)^{2}+\left(\frac{\partial \bar{v}}{\partial z}\right)^{2}} .
$$

Predictions and observations are in agreement. In the LES simulation of $R i$ no z-less limit is observed. Instead there is a plateau between 70 and $130 \mathrm{~m}$ where $R i \sim 0.2$ followed by a strong increase at the SBL-top.

\section{Conclusion}

We have used an LES model to analyze the observations and theory presented in N84. The first and major conclusion is that our analysis does not cast any doubts on the correctness of the data, or on the the validity of the local scaling concept. A recent paper from [3] comes to similar conclusions based on (much) more modelling results and recent field and laboratory experiments $[15,16]$.

Also the idea proposed in N84 to filter the data in order to remove larger scale flucutations or gravity waves apparently leads to better agreement between theory and experiment.

The data presented in N84 have a large uncertainty, partly due to the difficulties associated with measuring weak turbulence signals but also due to meso scale effects and other disturbances associated with the imperfect horizontal homogeneity of the terrain. Nevertheless the careful selection of the data and the fact that the surroundings of Cabauw are really flat (terrain height variation amount to less than $\pm 5 \mathrm{~m}$ within a radius of $30 \mathrm{~km}$ or so), makes that it is still, after two decades, a unique data set, referred to by many investigators (see e.g [4]).

There are only a few data on SBL turbulence. The Minnesota dataset was one of the first [7] and was also used in N84. Recently some more data became available $[8,18,21]$, but these studies often focussed on special phenomena in the SBL such as intermittency, gravity waves, inertial oscillation, katabatic flow or other heterogeneous effects.

An interesting study of Ohya concerns a wind tunnel experiment of a laboratory SBL which would provide more accurate data obtained at lower Reynolds numbers.

Our data confirms the local scaling hypothesis proposed in N84, in line with the conclusions in [3]. For a stronger confirmation a number of LES should be performed in different atmospheric stability conditions and for a range of geostrophic wind forcings. We find in many cases (but not in all) a z-less scaling limit also in good agreement with the values reported here.

The temperature variance is an exception: the N84 observations show high uncertainties and have a large discrepancy with the Minnesota data. The N84 theory fails to describe both data sets. In fact, the LES simulation for the temperature variance comes closest to the (filtered) N84 data.

Having recognized the power of DNS simulation, Nieuwstadt re-investigated the dynamics of the stable boundary-layer shortly before his death, [14]. In that paper he addresses the decay of turbulence in an SBL, when the shear-generated turbulence is no longer able to sustain turbulent motion. Neglecting Coriolis forces, he shows that the flow, with Reynolds number $R e_{\star} \equiv u_{\star} h / v=360$, laminarizes for $h / L>1.25$. 
Here $u_{\star}$ denotes the friction velocity, $h$ the boundary-layer thickness, $v$ the molecular viscosity and $L$ a parameter which is proportional to the Obukhov length. Our simulations with small and constant $K_{m, h}$ are very similar to his, and we also have observed laminarization phenomena. (Our model operates with comparable values for $R e_{\star}=u_{\star} h / K_{m}, h=480$ and $h / L=1.8$ ). However, we think (see the end of Section 2) that a third parameter, $\Delta / h$, (also noted by Nieuwstadt) influences the laminarization process considerably. It would be interesting to further investigate the stability properties of DNS simulations in the parameter space $h / L, \Delta / h$ and $R e_{\star}$.

Nieuwstadt's experiment and theory of the SBL were a major achievement and an important contribution to our understanding of the SBL. They led to a unique article and served as a standard in many publications up to now.

\section{References}

1. Andrén, A.: The structure of stably stratified atmospheric boundary lyers: a large-eddy simulation study. Quart. J. Roy. Meteorol. Soc. 121, 961-985 (1995)

2. Asselin, R.: Frequency filter for time integration. Mon. Weather Rev. 100, 487-490 (1972)

3. Basu, S., Porté-Agel, F., Foufoula-Georgiou, E., Vinuesa, J.-F., Pahlow, M.: Revisiting the local scaling hypothesis in stably straified atmospheric boundary-layer turbulence: an integration of field and laboratory measurements with large-eddy simulations. Boundary - Layer Meteorol. 999, 1-28 (2006)

4. Beare, R.J., MacVean, M.K.: Resolution sensitivity and scaling of large-eddy simulations of the stable boundary layer. Boundary - Layer Meteorol. 112, 257-281 (2004)

5. Beare, R. J. et al.: An intercomparison of large-eddy simulations of the stable boundary layer. Boundary - Layer Meteorol. 118, 1-32 (2006)

6. Brost, R., Wyngaard, J.: A model study of the stably stratified planetary boundary layer. J. Atmos. Sci. 35, 1427-1440 (1978)

7. Caughey, S.J., Wyngaard, J., Kaimal, J.: Turbulence in the evolving stable boundary layer. J. Atmos. Sci. 36, 1041-1052 (1979)

8. Cuxart, J. et al.: Stable atmospheric boundary-layer experiment in Spain (sables98): a report. Boundary - Layer Meteorol. 96, 337-370 (2000)

9. Garratt, J.R.: The Atmospheric Boundary Layer, pp. 316. Cambridge Univ. Press (1992)

10. Holtslag, A.: Scaling the atmospheric boundary-layer. GEWEX news 13, 7-8 (2003)

11. Hunt, J.C.R., Stretch, D.D., Britter, R.E.: Length scales in stably stratified turbulent flows and their use in turbulence models, pp. 285-321. Clarendon Press (1988)

12. Mason, P.J., Derbyshire, S.H.: Large-eddy simulation of the stably-stratified atmospheric boundary layer. Boundary - Layer Meteorol. 53, 117-162 (1990)

13. Nieuwstadt, F.: The turbulent structure of the stable nocturnal boundary layer. J. Atmos. Sci. 41, 2202-2216 (1984)

14. Nieuwstadt, F.T.M.: Direct numerical simulation of stable channel flow at large stability. Boundary - Layer Meteorol. 116, 277-299 (2005)

15. Ohya, Y.: Wind-tunnel study of atmospheric stable boundary layers over a rough surface. Boundary - Layer Meteorol. 98, 57-82 (2001)

16. Pahlow, M., ad F. Porté-Agel, M.B.P.: On Monin-Obukhov similarity in the stable atmospheric boundary layer. Boundary - Layer Meteorol. 99, 225-248 (2001)

17. Piacsek, S., Williams, G.: Conservation properties of convective difference schemes. J. Comput. Phys. 6, 392-405 (1970)

18. Poulos, S.G. et al.: Cases-99: a comprehensive investigation of the stable nocturnal boundary layer. Bull. Am. Meteorol. Soc. 83, 555-581 (2002)

19. Schumann, U.: Subgrid scale model for finite difference simulations of turbulent flows in plane channels and annuli. J. Comput. Phys. 18, 376-404 (1975)

20. Siebesma, A.P., Cuijpers, J.W.M.: Evaluation of parametric assumptions for shallow cumulus convection. J. Atmos. Sci. 52, 650-666 (1995) 
21. van de Wiel, B.: Intermittent turbulence and oscillations in the stable boundary layer over land. Ph.D. thesis, Utrecht University, The Netherlands (2002)

22. Wyngaard, J.: Modelling the planetary boundary layer-extension to the stable case. Boundary Layer Meteorol. 9, 441-460 (1975)

23. van Zanten, M.C.: Entrainment processes in stratocumulus. Ph.D. thesis, Utrecht University (2000) 